\title{
Risk factors affect success rate of debridement, antibiotics and implant retention (DAIR) in periprosthetic joint infection
}

\author{
Yihong $\mathrm{Xu}^{\dagger}$, Liping Wang ${ }^{\dagger}$ and Weidong $\mathrm{Xu}^{*}$ (B)
}

\begin{abstract}
Background: Periprosthetic joint infection (PJI) is the most devastating complication of joint replacement that seriously affects the quality of life and causes a heavy burden to the families and society. Due to shorter hospital stays, lower costs, improved joint function and less morbidity, a process of debridement, antibiotics and implant retention (DAIR) is recommended as the preferred treatment for acute periprosthetic joint infection. However, the factors that impact the success rate of DAIR remain controversial. This article evaluates the influential factors of DAIR and provides insights for orthopaedics surgeons to make optimal decisions to improve the success rate of DAIR.

Conclusion: The poor general condition of patients, high preoperative C-reactive protein (CRP) level, repeated joint surgeries, and Methicillin-resistant Staphylococcus aureus (MRSA) infections may be associated with lower DAIR success rate. To the contrary, early surgery, radical debridement, exchange of removable components, washing with iodine and vacuum sealing drainage (VSD) may improve the success rate of DAIR. A sinus tract may not be absolutely contraindicated, but surgeons should treat it with caution. As there is no consensus on many issues, more high-quality research is required.
\end{abstract}

Keywords: Risk factors, Success rate, DAIR, PJI

\section{Introduction}

PJI is the most serious complication that contributes to more than a quarter of revision surgeries after joint arthroplasty $[1,2]$. As people pay more attention to the PJI, the infection rate following arthroplasty is decreasing. However, the increase in the number of patients with prosthetic joints in recent decades has added to the absolute number of PJI cases [3, 4]. Recently, a review showed that, with more arthroplasty cases, the cost of revision surgery for infection has put greater pressure on healthcare budgets [5].

\footnotetext{
* Correspondence: xuwdshanghai@126.com

${ }^{\dagger}$ Yihong $\mathrm{Xu}$ and Liping Wang contributed equally to this work.

Department of Osteoarthropathy Surgery, Changhai Hospital, Shanghai 200433, China
}

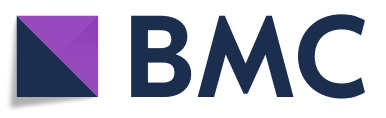

(c) The Author(s). 2020 Open Access This article is licensed under a Creative Commons Attribution 4.0 International License, which permits use, sharing, adaptation, distribution and reproduction in any medium or format, as long as you give

appropriate credit to the original author(s) and the source, provide a link to the Creative Commons licence, and indicate if changes were made. The images or other third party material in this article are included in the article's Creative Commons licence, unless indicated otherwise in a credit line to the material. If material is not included in the article's Creative Commons licence and your intended use is not permitted by statutory regulation or exceeds the permitted use, you will need to obtain permission directly from the copyright holder. To view a copy of this licence, visit http://creativecommons.org/licenses/by/4.0/. lieve pain and improve function. The therapeutic regimens include simple antibiotic treatment, DAIR, one-stage revision or two-stage revision, arthrodesis and amputation $[2,6]$. Due to the presence of biofilm on the surface of the prosthesis, the success rate of antibiotic therapy alone is very low. This treatment is reserved for patients who refuse surgery, or who are unable to undergo surgery due to their clinical circumstances such as concomitant illness, fragility, or advanced age [7]. One- or two-stage revision arthroplasty often place a burden on patients and families because of its significant injury and high cost. Arthrodesis and amputation are usually the last choice in clinical practice because of serious impairment of joint function and quality of life. The accepted indications for arthrodesis or amputation 
include poor soft tissue conditions around the prosthesis and bone tissue defects, highly-resistant organisms, the patient's poor general condition and failure of revision arthroplasty [8].

DAIR is currently considered the preferred treatment for acute PJI due to its low trauma, satisfactory costeffectiveness and less impairment on joint function [2, 9, 10]. The infection remission rate of DAIR for hip and knee PJI ranges from 11.1 to $100 \%$ [9, 11]. However, improving the success rate of DAIR is still a challenge.

\section{Preoperative patient factors \\ Sinus tract}

DAIR is only suitable for those PJI patients with good conditions of bone and soft tissue in whom no sinus tract exists between the joint prosthesis and the skin [12]. Qasim et al [13] showed that the emergence of a sinus tract was a high-risk factor for a failed DAIR procedure. Once the sinus tract forms, the success rate of DAIR is reduced greatly. Some scholars suggest that the emergence of a sinus tract is an absolute contraindication for DAIR.

With the development of a surgical technique for DAIR and the application of postoperative antibiotics, some studies showed that the existence of a sinus tract was no longer an absolute contraindication for DAIR. A retrospective study reported 14 PJI cases of a sinus tract treated with radical debridement, removal of dead tissue, shaving of pseudo-membrane, and intraoperative irrigation with a large volume of physiological saline, hydrogen peroxide and iodine. A satisfactory outcome was achieved in all patients as there was no recurrence of infection after 1-5 years follow-up [14].

\section{Preoperative CRP level}

Preoperative CRP level is also an influential factor contributing to a failed outcome of DAIR [15]. Vilchez et al [16] believed that DAIR achieved better results in PJI patients when preoperative $\mathrm{CRP}<22 \mathrm{mg} / \mathrm{L}$. However, Maritinez Pastor et al [17] believed that only when the preoperative $\mathrm{CRP}<15 \mathrm{mg} / \mathrm{L}$ were outcomes better. Recently, a high-quality retrospective cohort study performed by Jacobs et al [18] reported that CRP level $>100 \mathrm{mg} / \mathrm{L}$ was associated with failure of DAIR. However, the cut-off value of CRP to help surgeons decide whether to perform the DAIR procedure cannot be determined at present. Therefore, the relationship between preoperative CRP level and the success rate of DAIR remains to be investigated further.

\section{Repeated joint surgery}

Previous joint surgery also affects the prognosis of DAIR. Abrman et al [19] reported that DAIR should be considered carefully in repeatedly operated joints. A multicenter cohort study performed by Peel et al [20] showed a higher risk of failure in cases of re-infection after revision surgery treated with DAIR.

\section{Other factors}

In addition to the above factors, age, immunity and nutritional conditions, the American Society of Anesthesiologists (ASA) score, and complications such as hypertension and diabetes also affect the success rate of DAIR [21].

There is no consensus on age. Although most scholars suggest increased age as a risk factor for failure, recently a high-quality meta-analysis [9] reported that the infection control rate was higher in older patients compared with younger patients. Moreover, a single-center retrospective study [22] reported that patients $<60$ years old at the time of DAIR had a greater than two-fold increased risk of failure. Contradictory results relating age and DAIR success rate may be ascribed to the heterogeneity of studies, and further high-quality studies are required on this issue.

\section{Microbiology}

Although a $100 \%$ success rate of DAIR for Staphylococcus infection has been reported [23], most studies reported a higher failure rate of DAIR when performed to treat Staphylococcus infection [11, 22, 24-26]. A multicenter observational study performed by Weston et al [22] reported a three-fold increased risk of failure after DAIR in cases of Staphylococcus infection. Wouthuyzen et al [25] showed that although the outcome of DAIR in the management of early acute PJI was satisfactory, its use in late acute PJI caused by Staphylococcus should be reconsidered. A recent study found that the success rate in patients treated with DAIR for Staphylococcus infection was $76.2 \%$ [26].

The success rate of DAIR for treating periprosthetic infection caused by Streptococcus is relatively high [27, 28]. Lam et al [27] reported that the initial success rate of DAIR in the treatment of 53 PJI cases caused by Streptococcus infection was $84 \%$. However, in 2017, a large multicenter retrospective study showed that the success rate of DAIR for Streptococcus infection was only 57.9\% [29].

Periprosthetic infections caused by gram-negative bacteria have been infrequently reported. A large multicenter retrospective observational study [30] reported a $79 \%$ success rate for gram-negative prosthetic joint infection treated with DAIR.

McArthur et al [31] reported 16 of 26 infections caused by organisms of low virulence such as Coagulasenegative Staphylococcus, Acinetobacter, Propionibacterium species, and Corynebacterium species which were more common in seronegative infections. This study 
suggested that low virulence infections may contribute to a relatively high success rate. However, the sample size was small and further data are needed.

\section{Timing of the surgery}

Fehring et al [32] showed a limited ability of DAIR to control infection in the early postoperative period following arthroplasty. Grammatopoulos et al [28] suggested DAIR be performed in all PJI cases, regardless of interval from the index symptoms. A similar PJI eradication rate was seen if DAIR was undertaken after six $(78 \%)$ or 13 weeks $(83 \%)$. However, most scholars regard the timing of DAIR as a key factor in its success rate in PJI patients. In general, DAIR is recommended for acute infection or acute hematogenous infection.

Sendi et al [33] reported a successful outcome rate in 91\% of 34 PJI cases after total hip arthroplasty (THA), the duration of symptoms did not exceed 21 days. Ottesen et al [34] reported that there was no obvious difference in success rate between DAIR performed within 28 $(85 \%)$ or 42 days $(88 \%)$. However, the success rate was significantly lower when performing DAIR after 90 days (60\%). A review of cohort studies that included 1296 patients [35] reported a statistically significantly greater success rate when DAIR took place at a median of less than 7 days from the onset of symptoms of infection compared with debridement performed at a median of 1 week after the onset of symptoms (72.0\% versus $51.8 \%$ ).

The presence of biofilm on the surface of an implant is the most important reason for the transformation of an early infection of an artificial joint into a chronic infection [36]. Three weeks after infection, pathogenic microorganisms form a membranous structure on the surface of the prosthesis and enter the maturation stage. At this point, even open debridement can't remove the biofilm and bacteria sessiled in the biofilm completely. Meanwhile, due to the continuous shedding of biofilm debris, bacteria in the biofilm become plankton, resulting in continuous and repeated infection. Above all, DAIR can be used to treat patients with acute PJI within 4 weeks of infection before the bacteria have been sessiled on the surface of the prosthesis and the biofilm matures. Therefore, we believe that DAIR should be performed as soon as possible after the identification of symptoms of acute infection to avoid the transformation of an acute infection into a chronic infection.

\section{Arthroscopic or open debridement}

Liu et al [37] reported an 88\% success rate in 15 patients treated by arthroscopic debridement and continuous antibiotic irrigation. Dixon et al [38] showed that arthroscopic debridement was more effective in patients with periprosthetic infection of the knee. Though findings of a meta-analysis [23] suggested a higher infection control rate for arthroscopic debridement compared with open debridement, the results were not significantly different. The pooled infection control rate for arthroscopic DAIR was based on only a few studies. More studies showed a lower success rate of arthroscopic DAIR when compared to open debridement $[39,40]$. We suggest the DAIR procedure cannot be carried out arthroscopically because it does not allow adequate debridement or exchange of the polyethylene liner.

\section{Radical debridement}

Thorough debridement is essential to the success of DAIR. All infected tissues, synovial membrane, necrotic tissues, scar tissue and foreign materials must be completely removed. The success rate of DAIR in PJI patients is very low if debridement is not performed [41].

\section{Exchange of removable components}

The prosthesis is retained when DAIR is performed to treat PJI, but removable components must be exchanged whenever possible [13, 28, 35, 42]. T Sang et al [35] reported a mean success rate of $73.9 \%$ in PJI patients who underwent exchange of modular components (femoral head or acetabular liner) at the time of debridement. This was higher than in patients for whom no components were exchanged (60.7\%). Recently, a single-center retrospective cohort study performed by Hirsiger et al [42] showed that the exchange of mobile components of the PJI during DAIR almost doubled the long-term remission rate compared to the control group. Almost all removable components are made of polyethylene to which bacteria can easily adhere and some bacteria remain in the dead corner and the back of the liner after debridement. Only by exchanging components can we debride the infection thoroughly. Once the components are retained during debridement, the infection may recur easily and this ultimately can lead to the failure of treatment.

\section{Washing with povidone-iodine}

High volume povidone-iodine and low-pressure irrigation should be used intraoperatively with a lavage volume at least $9 \mathrm{~L}$. Li et al [43] reported that the success rate of washing with iodine in DAIR was $89.3 \%$. They suggested that for patients with PJI within 4 weeks, DAIR could be performed. When the prosthesis was soaked with iodine four times intraoperatively, the curative effect was better than conventional debridement. High-volume irrigation of povidone-iodine intraoperatively similarly was recommended by Choo KJ et al [44]

\section{Vacuum sealing drainage (VSD)}

VSD helps to continuously drain necrotic tissue and exudate, eliminate edema, stimulate growth of the 
granulation tissue, improve local blood supply and enhance local immunity. Since its development, the VSD technique has been applied to the treatment of PJI with increasing frequency.

The negative pressure value of VSD also had a significant influence on the effect of drainage. When high negative pressure drainage was performed, the total drainage and infection control rate were significantly better compared to low negative pressure drainage [45]. In 2014, Anagnostakos et al [46] reported that the success rate of DAIR combined with VSD was as high as $92.8 \%$.

We suggest VSD be used for selected patients because of limitations such as requirement for frequent exchange of materials which increases hospitalization expenses and hospital stays.

\section{Single or multiple debridement}

A voluminous literature discussed the relationship between single or multiple debridement and the success rate of DAIR. Some scholars reported that multiple debridement was related to the increased failure rate of DAIR [42, 47-50]. Estes et al [47] reported that 18 of 20 PJI patients utilizing a two-stage debridement technique with the implantation of antibiotic cement beads in stage one achieved a satisfactory outcome. Geurts et al [48] also reported good results for deep infections following total hip or knee arthroplasty with multiple debridement. Romano et al [49] reported that the success rate of multiple debridement was higher than that of single debridement. Chung AS et al [50] reported that 2-stage debridement led to a greater likelihood of infection control compared to single stage debridement. In contrast, a case control study by Moojen et al [51] reported a retrospective comparative study between single debridement and multiple debridement, finding no statistically significant difference in the proportion of successful outcomes between the two groups ( $88 \%$ vs. $71 \%)$. Moreover, Jacobs et al [18] found that multiple debridement procedures contributed to failed DAIR due to the risk of joint and wound contamination during the surgery. As there is no consensus, we suggest that orthopedists should be meticulous when performing multiple debridement, considering the length of the hospital stay, cost and morbidity associated with failed DAIR.

\section{Postoperative antibiotics}

When PJI patients were treated by DAIR without postoperative antibiotics, the failure rate after 3 years followup was as high as $86 \%$ [36].

For PJI patients with positive bacterial culture and drug sensitivity test results, antibiotics sensitive to pathogenic bacteria should be used after DAIR. The selected antibiotics also should be biofilm- penetrative to kill the pathogenic bacteria remaining in the biofilm after DAIR.

For PJI patients with negative bacteria culture or no drug sensitivity results, empirical antibiotic therapy usually is required. Selected antibiotics must have an adequately broad antibacterial spectrum to cover common pathogenic microorganisms. Sousa et al [52] suggested that vancomycin could be selected for patients with culture-negative PJI. However, Aboltins et al [53] reported that postoperative use of rifampicin combined with ciprofloxacin for culture-negative PJI led to a good outcome. The international consensus on PJI published by Parvizi et al [54] in 2013 suggested that vancomycin should be used for gram-positive bacteria and gentamicin or the third and fourth generation cephalosporin should be used for gram-negative bacteria in areas where MRSA is prevalent. However, for areas with low MRSA prevalence, the empirical use of vancomycin was not recommended until the result of drug sensitivity is available.

As to the duration of antibiotic treatment after DAIR, the Infectious Diseases Society of America (IDSA) advises initial intravenous therapy for 2-6 weeks followed by oral antibiotics for 3 months after hip surgery or 6 months after knee surgery. Several studies showed no differences in infection control rate when comparing shorter duration with longer duration postoperative antibiotics. Other studies indicate that prolonged parenteral antibiotic therapy increases the economic burden and the risk of drug resistance $[55,56]$.

\section{Conclusion}

DAIR is the preferred treatment for acute PJI with lower trauma and cost compared to revision surgery. A poor general condition of the patient, high preoperative CRP level, repeated joint surgery, MRSA infections may be associated with reduced DAIR success rate. Early surgery, radical debridement, the exchange of removable components, washing with iodine and VSD may improve the success rate of DAIR. A sinus tract may not absolutely contraindicate DAIR, but surgeons should treat it with caution. Orthopedic surgeons should take precautions to achieve a better outcome in PJI patients. As studies about DAIR are mostly retrospective and involve small samples, there is still no consensus on many aspects of DAIR effectiveness. More high-quality researches are required to improve the success rate of DAIR for PJI.

\section{Abbreviations}

PJI: Periprosthetic joint infection; DAIR: Debridement, antibiotics and implant retention; CRP: C-reactive protein; ASA: American Society of

Anesthesiologists; MRSA: Methicillin-resistant Staphylococcus aureus; THA: Total hip arthroplasty; VSD: Vacuum sealing drainage; IDSA: Infectious Diseases Society of America 


\section{Acknowledgements}

Not applicable.

\section{Authors' contributions}

Yihong Xu searched literature and drafted the manuscript. Liping Wang helped to draft the manuscript. Weidong Xu developed the idea for the study and revised the manuscript. All authors read and approved the final manuscript.

\section{Funding}

The authors declare that they received no funding from anyone or any institution.

\section{Availability of data and materials}

Data sharing not applicable to this article as no datasets were generated or analyzed during the current study.

\section{Ethics approval and consent to participate}

Not applicable.

\section{Consent for publication}

Not applicable.

\section{Competing interests}

The authors declare that they have no competing interests.

Received: 13 July 2020 Accepted: 27 October 2020

Published online: 07 December 2020

\section{References}

1. Lenguerrand $E$, Whitehouse MR, Beswick AD, et al. Risk factors associated with revision for prosthetic joint infection following knee replacement: an observational cohort study from England and Wales. Lancet Infect Dis. 2019; 19(6):589-600.

2. Kapadia BH, Berg RA, Daley JA, Fritz J, Bhave A, Mont MA. Periprosthetic joint infection. Lancet. 2016:387(10016):386-94.

3. Tande AJ, Patel R. Prosthetic joint infection. Clin Microbiol Rev. 2014;27: 302-45.

4. Saconi ES, de Carvalho VC, de Oliveira PRD, Lima ALLM. Prosthetic joint infection due to Candida species: case series and review of literature. Medicine (Baltimore). 2020;99(15):e19735.

5. Haddad FS, Ngu A, Negus JJ. Prosthetic joint infections and cost analysis? Adv Exp Med Biol. 2017;971:93-100

6. Ahmed SS, Begum F, Kayani B, Haddad FS. Risk factors, diagnosis and management of prosthetic joint infection after total hip arthroplasty. Expert Rev Med Devices. 2019;16(12):1063-70.

7. Chen AF, Heller S, Parvizi J. Prosthetic joint infections. Surg Clin North Am. 2014;94(6):1265-81.

8. Fagotti L, Tatka J, Salles MJC, Queiroz MC. Risk factors and treatment options for failure of a two-stage exchange. Curr Rev Musculoskelet Med. 2018;11(3):420-7.

9. Kunutsor SK, Beswick AD, Whitehouse MR, Wylde V, Blom AW. Debridement, antibiotics and implant retention for periprosthetic joint infections: a systematic review and meta-analysis of treatment outcomes. J Inf Secur. 2018;77(6):479-88.

10. Barros LH, Barbosa TA, Esteves J, Abreu M, Soares D, Sousa R. Early debridement, antibiotics and implant retention (DAIR) in patients with suspected acute infection after hip or knee arthroplasty - safe, effective and without negative functional impact. J Bone Jt Infect. 2019;4(6):300-5.

11. Urish $\mathrm{KL}$, Bullock $\mathrm{AG}$, Kreger $\mathrm{AM}$, et al. A multicenter study of irrigation and debridement in total knee arthroplasty periprosthetic joint infection: treatment failure is high. J Arthroplast. 2018;33(4):1154-9,

12. Zimmerli W, Trampuz A, Ochsner PE. Prosthetic joint infections. N Engl J Med. 2004;351(16):1645-54

13. Qasim SN, Swann A, Ashford R. The DAIR (debridement, antibiotics and implant retention) procedure for infected total knee replacement - a literature review. SICOT J. 2017:3:2

14. Shao Z, Zhang $Y, X u$ W, et al. Outcome analysis of surgical debridement and antibiotic treatment for prosthetic joint-associated infection with retention of implant. Chin J Bone Joint Inj. 2016;31(4):49-352.
15. Lora Tamayo J, Murillo O, Iribarren JA, et al. A large multicenter study of methicillin susceptible and methicillin resistant Staphylococcus aureus prosthetic joint infection managed with implant retention. Clin Infect Dis. 2013;56(2):182-94

16. Vilchez F, Martinez Pastor JC, Garcia Ramiro S, et al. Outcome and predictors of treatment failure in early post surgical prosthetic joint infections due to Staphylococcus aureus treated with debridement. Clin Microbiol Infect. 2011;17(3):439-44.

17. Martinez Pastor JC, Munoz-Mahamud E, Vilchez F, et al. Outcomes of acute prosthetic joint infections due to gram negative bacilli treated with open debridement and retention of the prothesis. Antimicrob Agents Chemother. 2009:53(11):4772-7.

18. Jacobs AME, Valkering LJ, Bénard M, Meis JF, Goosen JHM. Evaluation one year after DAIR treatment in 91 suspected early prosthetic joint infections in primary knee and hip Arthroplasty. J Bone Jt Infect. 2019:4(5):238-44.

19. Abrman K, Musil D, Stehlík J. Treatment of acute periprosthetic infections with DAIR (debridement, antibiotics and implant retention) success rate and risk factors of failure. Acta Chir Orthop Traumatol Cechoslov. 2019;86(3):181-7.

20. Tn P, Cheng AC, Choong PF, et al. Early onset prosthetic hip and knee joint infection: treatment and outcomes in Victoria, Australia. J Hosp Infect. 2012; 82(4):248-53.

21. Buller LT, Sabry FY, Easton RW, et al. The preoperative prediction of success following irrigation and debridement with polyethylene exchange for hip and knee prosthetic joint infections. J Arthroplast. 2012;27(6):857-64.

22. Weston JT, Watts CT, Mabry TM, et al. Irrigation and debridement with chronic antibiotic suppression for the management of infected total knee arthroplasty: a contemporary analysis. Bone Joint J. 2018;100-B:1471-6.

23. Zhang CF, He L, Fang XY, et al. Debridement, antibiotics, and implant retention for acute periprosthetic joint infection. Orthop Surg. 2020; 12(2):463-70

24. Azzam KA, Seeley M, Ghanem E, et al. Irrigation and debridement in the management of prosthetic joint infection: traditional indications revisited. J Arthroplast. 2010;25(7):1022-7.

25. Wouthuyzen-Bakker M, Sebillotte M, Huotari K, et al. Lower success rate of débridement and implant retention in late acute versus early acute periprosthetic joint infection caused by Staphylococcus spp. results from a matched cohort study. Clin Orthop Relat Res. 2020:478(6):1348-55.

26. Lesens $\mathrm{O}$, Ferry $\mathrm{T}$, Forestier $\mathrm{E}$, et al. Should we expand the indications for the DAIR (debridement, antibiotic therapy, and implant retention) procedure for Staphylococcus aureus prosthetic joint infections? A multicenter retrospective study. Eur J Clin Microbiol Infect Dis. 2018. 37(10):1949-56.

27. Lam A, Rasmussen M, Thompson O. Successful outcome for patients with streptococcal prosthetic joint infections - a retrospective population-based study. Infect Dis (Lond). 2018:50(8):593-600.

28. Grammatopoulos G, DPhil, FRCS (T\&O)*, Kendrick B, et al. Outcome Following Debridement, Antibiotics, and Implant Retention in Hip Periprosthetic Joint Infection—An 18-Year Experience. J Arthroplast. 2017; 32(7):2248-55

29. Lora-Tamayo J, Senneville É, Ribera A, et al. The not-so-good prognosis of streptococcal periprosthetic joint infection managed by implant retention: the results of a large multicenter study. Clin Infect Dis. 2017:64(12):1742-52.

30. Rodriguez Pardo D, Pigrau C, Lora Tamayo J, et al. Gram negative prosthetic joint infection: outcome of a debridement, antibiotic and implant retention approach. A large multicenter study. Clin Microbiol Infect. 2014;20(11):0911-9.

31. McArthur BA, Abdel MP, Taunton MJ, Osmon DR, Hanssen AD. Seronegative infections in hip and knee arthroplasty: periprosthetic infections with normal erythrocyte sedimentation rate and C-reactive protein level. Bone Joint J. 2015:97-B:939-44.

32. Fehring $T K$, Odum $S M$, Berend KR, et al. Failure of irrigation and debridement for early postoperative periprosthetic infection. Clin Orthop Relate Res. 2013;471(1):250-7.

33. Sendi P, Lötscher PO, Kessler B, Graber P, Zimmerli W, Clauss M. Debridement and implant retention in the management of hip periprosthetic joint infection: outcomes following guided and rapid treatment at a single Centre. Bone Joint J. 2017;99-B(3):330-6.

34 Ottesen CS, Troelsen A, Sandholdt H, Jacobsen S, Husted H, Gromov K. Acceptable success rate in patients with Periprosthetic knee joint infection treated with debridement, antibiotics, and implant retention. J Arthroplast. 2019;34(2):365-8. 
35. Tsang SJ, Ting J, Simpson AHRW, Gaston P, et al. Outcomes following debridement, antibiotics and implant retention in the management of periprosthetic infections of the hip: a review of cohort studies. Bone Joint J. 2017:99-B(11):1458-66.

36. Costerton JW. Biofilm theory can guide the treatment of device related orthopedic infections. Clin Orthop Relate Res. 2005:437:7-11.

37. Liu CW, Kuo CL, Chuang SY, Chang JH, Wu CC, Tsai TY, Lin LC. Results of infected total knee arthroplasty treated with arthroscopic debridement and continuous antibiotic irrigation system Indian. J Orthop. 2013;47(1):93-7.

38. Dixon P, Parish EN, Cross MJ. Arthroscopic debridement in the treatment of the infected total knee replacement. J Bone Joint Surg Br. 2004;86(1):39-42.

39. Waldman BJ, Hostin E, Mont MA, Hungerford DS. Infected total knee arthroplasty treated by arthroscopic irrigation and débridement. J Arthroplast. 2000;15(4):430-6.

40. Chung JY, Ha CW, Park YB, Song YJ, Yu KS. Arthroscopic debridement for acutely infected prosthetic knee: any role for infection control and prosthesis salvage? Arthroscopy. 2014;30(5):599-606.

41. Giulieri SG, Graber P, Ochsner PE, et al. Management of infection associated with total hip arthroplasty according to a treatment algorithm. Infection. 2004;32(4):222-8.

42. Hirsiger S, Betz M, Stafylakis D, Götschi T, Lew D, Uçkay I. The benefice of mobile parts' exchange in the management of infected total joint arthroplasties with prosthesis retention (DAIR procedure). J Clin Med. 2019;8(2):226

43. Li J, Shao Z, Tong W, et al. Clinical experience of implant retention in prosthetic joint infections with local application of povidone iodine. Chin J Joint Surg (Electronic Edition). 2017:11(4):421-6.

44. Choo KJ, Austin M, Parvizi J. Irrigation and debridement, modular exchange, and implant retention for acute periprosthetic infection after total knee arthroplasty. JBJS Essent Surg Tech. 2019;9(4):e38.1-2.

45. Zhao A, Guo X, Shi F. Application of closed negative pressure drainage device in treatment of early infections after hip arthroplasty. Chin J Nosocomiol. 2013;23(16):3954-6.

46. Konstantinos A. Cornelia et al. can periprosthetic hip joint infections be successfully managed by debridement and prosthesis retention? World $J$ Orthop. 2014;5(3):218-24.

47. Estes CS, Beauchamp CP, Clarke HD, et al. A two-stage retention Débridement protocol for acute periprosthetic joint infections. Clin Orthop Relat Res. 2010;468:2029-38.

48. Geurts JA, Janssen DM, Kessels AG, Walenkamp GH. Good results in postoperative and hematogenous deep infections of 89 stable total hip and knee replacements with retention of prosthesis and local antibiotics. Acta Orthop. 2013;84(6):509-16.

49. Romano CL, Manzi G, Logoluso N, et al. Value of debridement and irrigation for the treatment of periprosthetic infections-a systematic review. Hip Int. 2012;22(Suppl 8):S19-24.

50. Chung AS, Niesen MC, Graber TJ, et al. Two-stage debridement with prosthesis retention for acute periprosthetic joint infections. J Arthroplast. 2019:34:1207-13.

51. Moojen DJF, Zwiers JH, Scholtes VAB, Verheyen CCPM, Poolman RW. Similar success rates for single and multiple debridement surgery for acute hip arthroplasty infection. Acta Orthop. 2014;85:383-8.

52. Sousa R, Pereira A, Massada M, et al. Empirical antibiotic therapy in prosthetic joint infections. Acta Orthop Belg. 2010;76(2):254-9.

53. Aboltins C, Daffy J, Choong P, et al. Current concepts in the management of prosthetic joint infection. Intern Med J. 2014;44(9):834-40.

54. Parvizi J, Gehrke T, Chen AF. Proceedings of the international consensus on periprosthetic joint infection. Bone Joint J. 2013;95 B(11):1450-2.

55. Puhto AP, Puhto T, Syrjala H. Short-course antibiotics for prosthetic joint infections treated with prosthesis retention. Clin Microbiol Infect. 2012 18(11):1143-8.

56. Bernard L, Legout L, Zurcher-Pfund L, Stern R, Rohner P, Peter R, et al. Six weeks of antibiotic treatment is sufficient following surgery for septic arthroplasty. J Inf Secur. 2010;61(2):125-32.

\section{Publisher's Note}

Springer Nature remains neutral with regard to jurisdictional claims in published maps and institutional affiliations.

\section{Ready to submit your research? Choose BMC and benefit from:}

- fast, convenient online submission

- thorough peer review by experienced researchers in your field

- rapid publication on acceptance

- support for research data, including large and complex data types

- gold Open Access which fosters wider collaboration and increased citations

- maximum visibility for your research: over $100 \mathrm{M}$ website views per year

At $\mathrm{BMC}$, research is always in progress.

Learn more biomedcentral.com/submissions 